BULL. AUSTRAL. MATH. SOC.

VOL. $23(1981), 413-422$.

\title{
UNIFORMIZATION OF QUASI-UNIFORM SPACES
}

\author{
T.G. Raghavan and I.L. Reilly
}

\begin{abstract}
This paper considers the question of when a quasi-uniform space has a compatible uniform structure. Typical of the sufficient conditions provided is the result that a quasi-uniform space whose conjugate topology is compact and $R_{0}$ is uniformizable.
\end{abstract}

\section{Introduction}

Since Pervin [7] gave a simple direct proof of the fact that every topological space is quasi-uniformizable, there has been considerable interest in the study of quasi-uniform spaces. However, it seems that the question of when a quasi-uniform space is uniformizable has received very little attention. The purpose of this note is to provide some results in that direction.

It will be recalled that a quasi-uniformity on a set $X$ is a filter $U$ on $X \times X$ such that the diagonal $\Delta$ is contained in each member of $U$, and for each $U \in U$ there is a $V \in U$ such that $V \circ V \subset U$. If $U$ is a quasi-uniformity on $X$, then the topology $T(U)$ defined by the neighbourhood system $\{U[x]: x \in X\}$ is the topology induced by $U$, where $U[x]=\{U[x]: U \in U\}$. Each quasi-uniformity $U$ on $X$ induces $a$ conjugate quasi-uniformity $U^{-1}$ on $X$ defined by $u^{-1}=\left\{U^{-1}: U \in U\right\}$. Thus a quasi-uniformity $U$ on $X$ generates two topologies $T(U)$ and $T\left(U^{-1}\right)$ on $X$, and we are in the setting of bitopological spaces $\left(X, T_{1}, T_{2}\right)$ in the sense of Kelly [3], whose terms and notation we adopt

Received 24 December 1980. 
here. A bitopological space $\left(X, T_{1}, T_{2}\right)$ is quasi-uniformizable if there is a pair $U$ and $U^{-1}$ of conjugate quasi-uniformities on $X$ such that $T_{1}=T(U)$ and $T_{2}=T\left(U^{-1}\right)$, see Lane [4]. The bitopological space $\left(X, T_{1}, T_{2}\right)$ is pairwise completely regular if for each $T_{i}$ closed set $C$ and each point $x \leqslant C$ there is a real valued function $f: X \rightarrow[0,1]$ such that $f(x)=0, f(C)=\{1\}, f$ is $T_{i}$ upper semi-continuous and $T_{j}$ lower semi-continuous, for $i, j=1,2, i \neq j \cdot\left(X, T_{1}, T_{2}\right)$ is pairwise regular if for each $T_{i}$ closed set $C$ and each point $x \notin C$ there is a $T_{i}$ open set $U$ and a $T_{j}$ open set $V$ disjoint from $U$ such that $x \in U$ and $C \subset V$, for $i, j=1,2, i \neq j$.

In the bitopological space $\left(X, T_{1}, T_{2}\right)$ we say that

(i) $T_{1}$ is (countably) paracompact with respect to $T_{2}$ if each (countable) $T_{1}$ open cover of $X$ has a $T_{1}$ open refinement which is $T_{2}$ locally finite,

(ii) $T_{1}$ is locally (countably) compact with respect to $T_{2}$ if each point of $X$ has a $T_{2}$ neighbourhood which is $T_{1}$ (countably) compact.

A topological space $(X, T)$ is $R_{0}$ if it satisfies one of the following equivalent conditions:

$$
\begin{aligned}
& \text { (i) } x \in U \in T \text { implies } \operatorname{cl}\{x\} \subset U, \\
& \text { (ii) } \operatorname{cl}\{x\}=n\{U \mid U \text { is an open neighbourhood of } x\} \text { for each } \\
& x \in X, \\
& \text { (iii) } \operatorname{cl}\{x\}=\operatorname{cl}\{y\} \text { or } \operatorname{cl}\{x\} \cap \operatorname{cl}\{y\}=\emptyset \text { for } x, y \in X .
\end{aligned}
$$

The sufficiency conditions that we give for the uniformizability of $(x ; U)$ are in terms of the relationship between $T(u)$ and $T\left(u^{-1}\right)$, or solely conditions on the conjugate space. Our (quasi) uniform notation is standard and follows Murdeshwar and Naimpally [5].

We are not able to apply most of the results of Section 2 of [8] to 
the quasi-uniform situation because a quasi-uniformizable bitopological space is not necessarily pairwise Hausdorff. In Section 2 of this paper we prove similar results, but for considerably different hypotheses. In particular, the condition that one of the spaces be $R_{0}$ is crucial in the extension of our previous results for quasi-metric spaces to quasipseudometric spaces in Section 4.

\section{Bitopological results}

LEMMA 1. If $\left(X, T_{1}, T_{2}\right)$ is pairwise regular then $T_{i} \operatorname{cl}\{p\}=n\left\{U: U \in T_{j}\right.$ and $\left.p \in U\right\}$ for each point $p \in X$, where $i, j=1,2$ and $i \neq j$.

Proof. Let $p \in U \in T_{j}$. Then pairwise regularity gives a $V \in T_{j}$ such that $p \in V \in T_{i}$ cl $V \subset U$. If $B=\cap\left\{U: U \in T_{j}\right.$ and $\left.p \in U\right\}$, then $B=n\left\{T_{i}\right.$ cl $U: U \in T_{j}$ and $\left.p \in U\right\}$. Thus $B$ is $T_{i}$ closed, so that $T_{i} \operatorname{cl}\{p\} \subset B$.

Now suppose $q \in B-T_{i} c l\{p\}$. Then $L_{1}=X-T_{i}$ cl $\{p\}$ is $T_{i}$ open and $q \in L_{1}$. So there is, by pairwise regularity, an $L_{2} \in T_{i}$ such that $q \in L_{2} \subset T_{j}$ cl $L_{2} \subset L_{1}$. Thus $T_{j} c 1\{q\} \subset L_{1}$, so that $T_{j} \operatorname{cl}\{q\} \cap T_{i} \operatorname{cl}\{p\}=\varnothing$. Then if $U^{\prime}=X-T_{j} c l\{q\}$, we have $p \in U^{\prime} \in T_{j}$ and $q \notin U^{\prime}$, contradiction. Thus $T_{i} \operatorname{cl}\{p\}=B$.

LEMMA 2. Let $\left(X, T_{1}, T_{2}\right)$ be pairwise regular and $\left(X, T_{1}\right)$ be $R_{0}$. Then every $T_{1}$ closed $T_{1}$ compact subset of $X$ is $T_{2}$ closed.

Proof. Let $A \subset X$ be $T_{1}$ closed and $T_{1}$ compact. Then $x \in X-A$ implies $T_{1} \operatorname{cl}\{x\} \subset X-A$. By Lemma 1 ,

$$
T_{1} c l\{x\}=n\left\{U: U \in T_{2} \text { and } x \in U\right\}=n\left\{T_{1} \text { cl } U: U \in T_{2} \text { and } x \in U\right\} \text {. }
$$

Since $A \subset X-T_{1}$ cl $\{x\}$, we have that $\left\{X-T_{1}\right.$ cl $U: U \in T_{2}$ and $\left.x \in U\right\}$ is a $T_{1}$ open cover of $A$. Hence there is an integer $n$ and $T_{2}$ open sets $U_{1}, U_{2}, \ldots, U_{n}$ such that $A \subset U\left\{X-T_{1}\right.$ cl $\left.U_{k}: k=1,2, \ldots, n\right\}$ and 
$x \in V=\cap\left\{U_{k}: k=1,2, \ldots, n\right\}$. Thus $V$ is $T_{2}$ open and $V \cap A=\emptyset$, so that $A$ is $T_{2}$ closed.

PROPOSITION 1. If $\left(X, T_{1}, T_{2}\right)$ is pairwise regular, $\left(X, T_{1}\right)$ is $R_{0}$ and $T_{1}$ is locally compact with respect to $T_{2}$, then $T_{1} \subset T_{2}$.

Proof. Let $x \in P \in T_{1}$, and $A$ be a $T_{2}$ neighbourhood of $x$ which is $T_{1}$ compact. By pairwise regularity, there is a $Q \in T_{2}$ such that $x \in Q \subset T_{1}$ cl $Q \subset A$. Thus $T_{1}$ cl $Q$ is a $T_{2}$ neighbourhood of $x$ which is $T_{1}$ compact. Now let $S=Q-P$. Then $T_{1}$ cl $S$ is $T_{1}$ compact and $x \$ T_{1}$ cl $S$. By Lemma $2, T_{1}$ cl $S$ is $T_{2}$ closed, so that $V=X-T_{1}$ cl $S$ is $T_{2}$ open, contains $x$ and $V \cap S=\emptyset$. Then $U=Q \cap V$ is $T_{2}$ open and $x \in U \subset P$, so that $P \in T_{2}$.

PROPOSITION 2. If $\left(X, T_{1}, T_{2}\right)$ is pairwise regular, $\left(X, T_{1}\right)$ is $R_{0}$ and $T_{1}$ is paracompact with respect to $T_{2}$, then $T_{1} \subset T_{2}$.

Proof. Let $x \in V \in T_{1}$. Then $T_{1} \operatorname{cl}\{x\} \subset V$, and $T_{1} c l\{x\}=n\left\{U: U \in T_{2}\right.$ and $\left.x \in U\right\}=n\left\{T_{1}\right.$ cl $U: U \in T_{2}$ and $\left.x \in U\right\}$. Thus $X-T_{1} \operatorname{cl}\{x\}=U\left\{X-T_{1}\right.$ cl $U: U \in T_{2}$ and $\left.x \in U\right\}$, so that $\mathcal{C}=\left\{V,\left\{X-T_{1}\right.\right.$ cl $U: U \in T_{2}$ and $\left.\left.x \in U\right\}\right\}$ is a $T_{1}$ open cover of $X$. Now the proof follows that of Proposition 4 of [8].

COROLLARY 1. If $\left(X, T_{1}, T_{2}\right)$ is pairwise regular and $\left(X, T_{1}\right)$ is $R_{0}$ and compact, then $T_{1} \subset T_{2}$.

Proof. If $\left(X, T_{1}\right)$ is compact, then $T_{1}$ is paracompact with respect to $T_{2}$ for any topology $T_{2}$ on $X$.

The next four results show that we can weaken the compactness conditions in the previous three results to the corresponding countable compactness conditions provided that we add an additional hypothesis. We omit the proofs which are obvious modifications of those above. 
LEMMA 3. Let $\left(X, T_{1}, T_{2}\right)$ be pairwise regular, $\left(X, T_{1}\right)$ be $R_{0}$ and $\left(X, T_{2}\right)$ be first countable. Then every $T_{1}$ closed $T_{1}$ countably compact subset of $X$ is $T_{2}$ closed.

PROPOSITION 3. If $\left(X, T_{1}, T_{2}\right)$ is pairwise regular, $\left(X, T_{1}\right)$ is $R_{0},\left(X, T_{2}\right)$ is first countable and $T_{1}$ is locally countably compact with respect to $T_{2}$, then $T_{1} \subset T_{2}$.

PROPOSITION 4. If $\left(X, T_{1}, T_{2}\right)$ is pairwise regular, $\left(X, T_{1}\right)$ is $R_{0},\left(X, T_{2}\right)$ is first countable and $T_{1}$ is countably paracompact with respect to $T_{2}$, then $T_{1} \subset T_{2}$.

COROLLARY 2. If $\left(X, T_{1}, T_{2}\right)$ is pairwise regular, $\left(X, T_{1}\right)$ is $R_{0}$ and countably compact, and $\left(X, T_{2}\right)$ is first countable, then $T_{1} \subset T_{2}$.

Again, we can weaken the compactness conditions to corresponding Lindelof properties if we add an additional hypothesis. A topological space is called a $P$-space if each $G_{\delta}$ set is open.

LEMMA 4. Let $\left(X, T_{1}, T_{2}\right)$ be pairwise regular, $\left(X, T_{1}\right)$ be $R_{0}$ and $\left(X, T_{2}\right)$ be a P-space. Then every $T_{1}$ closed $T_{1}$ Lindelof subset of $X$ is $T_{2}$ closed.

PROPOSITION 5. If $\left(X, T_{1}, T_{2}\right)$ is pairwise regular, $\left(X, T_{1}\right)$ is $R_{0},\left(X, T_{2}\right)$ is a $P$-space and $T_{1}$ is Locally Lindelof with respect to $T_{2}$, then $T_{1} \subset T_{2}$.

PROPOSITION 6. If $\left(X, T_{1}, T_{2}\right)$ is pairwise regular, $\left(X, T_{1}\right)$ is $R_{0},\left(X, T_{2}\right)$ is a $P$-space and $T_{1}$ is paralindelof with respect to $T_{2}$, then $T_{1} \subset T_{2}$.

COROLLARY 3. If $\left(X, T_{1}, T_{2}\right)$ is pairwise regutar, $\left(X, T_{1}\right)$ is $R_{0}$ and Lindelof, and $\left(X, T_{2}\right)$ is a P-space, then $T_{1} \subset T_{2}$. 


\section{Quasi-uniform applications}

The characterization of quasi-uniformizable bitopological spaces in Lemma 5 was given independently by Fletcher [1] and Lane [4].

LEMMA 5. $\left(X, T_{1}, T_{2}\right)$ is quasi-uniformizable if and only if it is pairwise completely regular.

It follows immediately from Lemma 5 that if $\left(X, T_{1}, T_{2}\right)$ is quasiuniformizable then it is pairwise regular.

LEMMA 6. If $\left(X, T_{1}, T_{2}\right)$ is quasi-uniformizable and $T_{1} \subset T_{2}$ then $\left(X, T_{2}\right)$ is uniformizable.

Murdeshwar and Naimpaily [5, Theorem 1.47] proved Lemma 6 by showing that if $T_{1}=T(U)$ and $T_{2}=T\left(U^{-1}\right)$ and $T_{1} \subset T_{2}$ then the supremum $u v u^{-1}$ of $u$ and $u^{-1}$ is a uniformity which generates the topology $T_{2}$. A subbasis for $u \vee u^{-1}$ is $\left\{u, U^{-1}: U \in U\right\}$, and $u \vee u^{-1}$ is the smallest quasi-uniformity which is finer than both $u$ and $u^{-1}$.

Since the proofs of the results in this section use Lemma 6 to provide a uniformity compatible with the given quasi-uniform space in each case, the results are more than existence theorems. They are constructive results, in that the given quasi-uniform space is uniformizable by the supremum of the given quasi-uniformity and its conjugate.

THEOREM 1. Every quasi-uniform space whose conjugate topology is compact and $R_{0}$ is uniformizable.

Proof. This follows from Lemma 5, Corollary 1 and Lemma 6.

THEOREM 2. If $\left(X, T_{1}, T_{2}\right)$ is quasi-uniformizable and $\left(X, T_{1}\right)$ is $R_{0}$, then $\left(X, T_{2}\right)$ is uniformizable if either

(i) $T_{1}$ is locally compact with respect to $T_{2}$, or

(ii) $T_{1}$ is paracompact with respect to $T_{2}$.

Proof. (i) follows from Proposition 1 and Lemmas 5 and 6 , and (ii) 
from Proposition 2 and Lemmas 5 and 6.

Using Corollary 2 we can obtain

THEOREM 3. Every first countable quasi-uniform space whose conjugate topology is $R_{0}$ and countably compact is uniformizable.

Corollary 3 yields the following result.

THEOREM 4, Every quasi-uniform P-space whose conjugate topology is $R_{0}$ and Lindelof is uniformizable.

In each case there is an analogue of Theorem 2 which we do not state.

In view of the fact that $R_{0}$ is a conjugate invariant property [5, Theorem 5.1], the $R_{0}$ hypothesis in each of the four theorems above can be on the given quasi-uniform space rather than on its conjugate.

Now we provide an example to show that the $R_{0}$ condition in our results cannot be replaced by $T_{0}$. First we make some comments about Pervin's quasi-uniformity and its conjugate. If $(X, T)$ is a topological space, the family $\left\{S_{G}: G \in T\right\}$ of subsets of $X \times X$ generates a quasiuniformity $U$ on $X$ compatible with $T$, where $S_{G}=(G \times G) \cup(X-G) \times X$, since $S_{G}[x]=G$ if $x \in G$, and $X$ if $x \notin G$; see Pervin [7]. The conjugate $U^{-1}$ is generated by the family $\left\{S_{G}^{-1}: G \in T\right\}$, and $S_{G}^{-1}=(G \times G) \cup X \times(X-G)$. Furthermore, $S_{G}^{-1}[x]=X$ if $x \in G$, and $X-G$ if $x \notin G$.

EXAMPLE 1 . Let $X=\{a, b\}$ and $T_{1}=\{\varnothing, X,\{a\}\}$. Then $\left(X, T_{1}\right)$ is not uniformizable because it is not regular, since $\{b\}$ is $T_{1}$ closed and $a \notin\{b\}$ but they cannot be separated by disjoint $T_{1}$ open sets. Let $u$ be the Pervin quasi-uniformity for $T_{1}$ and $u^{-1}$ its conjugate. Then $T_{2}=T\left(U^{-1}\right)$ is defined by the neighbourhood system $\left\{X-G: G \in T_{1}\right\}$. Thus $\tau_{2}=\{x, \emptyset,\{b\}\}$. Hence $\left(X, \tau_{1}\right)$ is a non-uni formizable quasi-uniform space whose conjugate topology is $T_{0}$ and compact. 
Our second example shows that the compactness conditions in our results cannot be relaxed too far.

EXAMPLE 2 (Patty [6, Example 2.6]). Let $X$ be the set consisting of $\{1 / n: n$ a positive integer $\}$ together with two points 0 and $0^{\prime}$. Define the quasi-metric $p$ on $X \times X$ by $p(x, x)=0$ for each $x \in X$, $p(x, 0)=1$ if $x \neq 0, p\left(x, 0^{\prime}\right)=1$ if $x \neq 0^{\prime}$, $p(0,1 / n)=p\left(0^{\prime}, 1 / n\right)=1 / n$ and $p(1 / n, 1 / m)=|(1 / n)-(1 / m)|$. Let $u$ be the quasi-uniformity and $T_{1}$ the topology induced on $X$ by $p$. Define $q$ by $q(x, y)=p(y, x)$ for $x, y \in X$.

Then $q$ induces the conjugate quasi-uniformity $u^{-1}$ of $u$, and the topology $T_{2}$ on $X$. Then $(X, U)$ is a quasi-uniform space which is not uniformizable, because it is not Hausdorff and hence, being $T_{1}$, it is not completely regular. However, its conjugate topology $T_{2}$ is discrete and countable, and so has all the following properties: Lindelof, second countable, separable, paracompact, locally compact, $\sigma$-compact, metacompact, countably paracompact, and it is a $k$-space. Thus no combination of these properties can replace the compactness conditions in Theorems 1,2 and 3. Furthermore, $\left(X, T_{1}\right)$ is not a $P$-space but its conjugate topology is $R_{0}$ and Lindelof. Thus the $P$-space condition cannot be dropped from Theorem 4 .

\section{Quasi-pseudometric applications}

The following result was proved by Kelly [3, Proposition 4.2 and Lemma 4.3].

LEMMA 7. If $p$ and $q$ are a pair of conjugate quasi-pseudometrics on $X$ then

(i) $\left(X, T_{p}, T_{q}\right)$ is pairwise regular,

(ii) if $T_{p} \subset T_{q}$ then $T_{q}$ is pseudometrizable.

THEOREM 5. Every quasi-pseudometric space whose conjugate topology is $R_{0}$ and countably compact is pseudometrizable.

Proof. Follows from Lemma 7 and Corollary 2. 
We observe that Theorems 1 and 4 of [8] are immediate corollaries of Theorem 5, since any quasi-metric space is $T_{1}$ and therefore $R_{0}$. Similarly Theorems 2 and 3 of [8] follow immediately from the following resuit.

THEOREM 6. If $\left(X, T_{1}, T_{2}\right)$ is quasi-pseudometrizable, and $\left(X, T_{1}\right)$ is $R_{0}$, then $\left(X, T_{2}\right)$ is pseudometrizable if either

(i) $T_{1}$ is locally countably compact with respect to $T_{2}$, or

(ii) $T_{1}$ is countably paracompact with respect to $T_{2}$.

Proof. (i) follows from Lemma 7 and Proposition 3, and (ii) from Lemma 7 and Proposition 4.

The next example shows that the $R_{0}$ condition is crucial in these results.

EXAMPLE 3 . Let $X$ be the interval $[0,1]$, and define a quasipseudometric $p$ on $X \times X$ by

$$
p(x, y)= \begin{cases}x-y & \text { if } x \geq y, \\ 0 & \text { if } x<y .\end{cases}
$$

Then $p$ induces the topology on $X$ consisting of rays open to the right, and its conjugate $q$ induces the topology on $X$ consisting of rays open to the left. Each of these topologies is compact and $T_{0}$ but not $T_{1}$, and hence not regular, and therefore not pseudometrizable. Neither of these topologies is $R_{0}$.

\section{References}

[1] Peter Fletcher, "Pairwise uniform spaces", Abstract 65T-328, Notices Amer. Math. Soc. 12 (1965), 612.

[2] Peter Fletcher, Hughes B. Hoyle, III and C.W. Patty, "The comparison of topologies", Duke Math. J. 36 (1969), 325-331. 
[3] J.C. Kelly, "Bitopological spaces", Proc. London Math. Soc. (3) 13 (1963), 71-89.

[4] E.P.Lane, "Bitopological spaces and quasi-uniform spaces", Proc. London Math. Soc. (3) 17 (1967), 241-256.

[5] M.G. Murdeshwar and S.A. Naimpally, Quasi-uniform topological spaces (Noordhoff, Groningen, 1966).

[6] C.W. Patty, "Bitopological spaces", Duke Math. J. 34 (1967), 387-391.

[7] William J. Pervin, "Quasi-uniformization of topological spaces", Math. Ann. 147 (1962), 316-317.

[8] T.G. Raghavan and I.L. Reilly, "Metrizability of quasi-metric spaces", J. London Math. Soc. (2) 15 (1977), 169-172.

Department of Mathematics,

University of Auckland,

Private Bag,

Auckland,

New Zealand. 